\title{
La combinación de lo real y lo fantástico en $E l$ otro yo y Acaso irreparable de Mario Benedetti
}

\author{
Aya Mahmoud Abdel Latif \\ Dpto. de Lengua Española, Facultad de Al-Alsun, Universidad de Minia, \\ Egipto \\ Email: aya.mahmoud@mu.edu.eg
}

Mario Benedetti (Paso de los Toros, 1920 - Montevideo, 2009 ) is a short story writer par excellence. In addition, he was an outstanding poet, novelist, playwright, critic, and journalist in The Generation '45. The short story occupies a very special place in Benedetti's literary career, as it is known that he has real backstories where he can reflect his reality and that of his country, along with other fantastic ones. This paper proposes that Benedetti combines reality with fantasy in some of his short stories. Two themes are analyzed through which the mixture of reality and fantasy appears clearly; these are the double and the interrelation of life and death in two of his short stories: The Other Self, and Perhaps Irreparable, concentrating on showing how Benedetti can combine the resources of time and space in the approach to reality and fantasy. Some aspects of magical realism that appear in these tales are also examined.

Keywords Benedetti, alter ego, death, reality, fantasy

\section{Resumen}

Mario Benedetti (Paso de los Toros, 1920-Montevideo, 2009) fue cuentista por excelencia, además fue un destacado poeta, novelista, 
dramaturgo, crítico y periodista de la generación del 45. El cuento ocupa un lugar muy especial en la trayectoria literaria de Benedetti. Como es sabido, el autor tiene cuentos de fondo meramente real donde se refleja su realidad y la de su país, y otros muy fantásticos. En este trabajo proponemos que Benedetti combine en algunos cuentos la realidad con la fantasía. Analizamos dos temas mediante los cuales aparece claramente la mezcla de la realidad y la fantasía que son el doble y la interrelación de la vida y la muerte en dos cuentos: El otro yo, y Acaso irreparable, concentrándonos en mostrar cómo Benedetti combina los recursos del tiempo y el espacio en el planteamiento de la realidad y la fantasía. También examinamos cómo aparecen algunos aspectos del realismo mágico en dichos cuentos.

Palabras clave: Benedetti, el doble, la muerte, lo real, lo fantástico

\section{Introducción}

El cuento ocupa un lugar muy especial en la trayectoria literaria de Benedetti no sólo por su destreza estilística, sino también por su diversidad de los temas y las ideas expresadas en ello, ya sea directamente en los cuentos reales o indirectamente en los que incluyen imaginación y realidad.

A continuación, a través de este trabajo pretendemos mostrar cómo Benedetti combina la realidad y la fantasía en los dos cuentos anteriormente mencionados utilizando varios recursos estilísticos considerando que el cuento en el que se mezcla lo real y lo fantástico no solo comunica un placer estético, sino que es un notable instrumento de indagación en la realidad, un método de conocimiento sobre hombres y épocas históricas y, a veces, afanador por la búsqueda del ser auténtico de los hombres en sí mismos, en su relación con los demás, con sus dudas y confusiones.

En el presente trabajo realizamos un análisis de los cuentos desde la perspectiva del realismo mágico y el realismo fantástico. A pesar de que algunos creen que son muy diferentes, pero pensamos que hay algunos rasgos comunes entre ambos, por ejemplo, la distracción del tiempo. Teniendo en cuenta que los dos se caracterizan sustancialmente por una 
combinación de la realidad y la fantasía. En este trabajo nos concentramos en destacar la ausencia de los límites entre lo real y lo ficticio en dichos cuentos.

\section{El doble}

El tema del doble es muy complejo y ha dado lugar a diferentes planteamientos en la literatura fantástica y ha intentado revelar el misterio de la identidad incomprensible del hombre cuya conciencia se siente desdoblada, insatisfecha o escindida. Ese misterio ha atraído la atención no solo de muchos filósofos, pensadores, sino de muchos poetas y escritores, Herrero Cecilia (2000, p. 252) propone que esto sucede debido a que:

La aparición del doble choca con los esquemas racionales (principio de no contradicción), constituye una anomalía o perturbación en el estado de cosas de la realidad ordinaria, y puede ser el signo de una fragmentación o escisión de la personalidad del individuo. Por eso el doble produce inquietud, sorpresa y fascinación en la mente del sujeto que percibe el misterioso fenómeno de duplicidad.

El tema del doble ha sido estudiado detalladamente y reflexionado durante todo el siglo XX, como hemos dicho no solo desde la visión de los filósofos, sino también, desde la perspectiva de la crítica literaria. Con el desarrollo de las teorías existencialistas y psicológicas, se empieza a construir un concepto del ser humano basado en la multiplicidad, en contra del concepto de la identidad del ser como algo estable, único e inamovible.

Romero (2008, p.476) arguye en su tesis doctoral los valores o los sentidos que podrían surgir utilizando el desdoblamiento:

en general, el desdoblamiento sirve para explicitar el conflicto del personaje, ya se trate de un conflicto interno o de un conflicto con otras personas que suelen pertenecer a su círculo familiar. Es, por tanto, un recurso que suele afectar a un personaje en conflicto consigo mismo o con los otros. A partir de ahí, el desdoblamiento es en ocasiones una fuente de conocimiento y de indagación en la otra entidad desdoblada. Otras veces el desdoblamiento es una vía para evadirse de una realidad prosaica y difícil. En otras ocasiones, el desdoblamiento enfrenta facetas opuestas y contradictorias de un mismo personaje, mostrando así la complejidad del espíritu humano. [...] el desdoblamiento supone contraste, oposición o enfrentamiento, por un lado, y por otro, también tiene matices de complementariedad, de alternativa, 
representando simbólicamente una faceta oculta, pero necesaria, del personaje.

Márquez (1994, citado por Romero, 2008, p. 476) se refiere a otro aspecto diferente que podría ser más positivo de la relación de contrastede la existencia de dos Yo:

la relación entre el yo y el otro surge precisamente como necesidad o deseo de Otro que le falta al Yo. Las causas de esa necesidad estarían en la pérdida, a la que asistimos en la época contemporánea, de la figura del otro por el triunfo de una cultura de la individualidad.

El cuento El otro yo del libro de cuentos La muerte y otras sorpresas, escrita en 1968, inicia con la descripción del protagonista de manera normal, concentrando en demostrar que Armando es una persona corriente. Además, expone varias de sus conductas humanas, donde encontramosreferencia a algunos malos hábitos. Hasta aquí se podría decir que tratamos con un cuento real: "Se trataba de un muchacho corriente: en los pantalones se le formaban rodilleras, leía historietas, hacía ruido cuando comía, se metía los dedos a la nariz, roncaba en la siesta, se llamaba Armando" (Benedetti, 2015, p. 255).

A partir de aquí, el carácter real del cuento empieza a experimentar un choque fantástico con la aparición del Otro Yo "Corriente en todo menos en una cosa: tenía Otro Yo. El Otro Yo usaba cierta poesía en la mirada, se enamoraba de las actrices, mentía cautelosamente, se emocionaba en los atardeceres" (Benedetti, 2015, p. 255). Puesto que el cuento revela la dualidad de su personalidad, lo que forma el rasgo fantástico y sobrenatural de este cuento donde el protagonista supone el núcleo de lo fantástico.

El narrador intensifica el significado de que la única diferencia de los demás es que posee Otro Yo. El conflicto interno surge básicamente porque está a disgusto con su Otro Yo. No parece normal que el desdoblamiento contraviene la base del individuo no solo desde la perspectiva psicológica, sino también morfológicamente. El origen de la palabra es "dividuo" del verbo dividir que significa, según el DRAE, partir o separar algo en partes, distribuir o repartir algo entre varios y averiguar cuántas veces una cantidad, llamada dividendo, contiene a otra, llamada divisor viene antes de esta raíz el prefijo "in" que indica 
negación o privación, es decir, expresa el valor contrario a la palabra que acompaña. Así, individuo significa que no se puede ser dividido, lo que contrasta con la capacidad del desdoblamiento de resultarse muchos en la personalidad.

El narrador no está satisfecho con su descripción, sino que también muestra una diferencia en los comportamientos de ambos que se llevan a cabo al mismo tiempo. Armando fue a dormir y deja el Otro escuchando música de Mozart. Por eso, El Otro Yo fue psicológicamente afectado por la música.

Al despertar, lo encontró llorando, lo que ha llevado a su fuerte reprimenda con la esperanza de deshacerse de su sensible lado infeliz debido a que no quería tener una conciencia tan humanista, y por lo tanto decide arrancarlo de sí mismo. Esto resultó en el suicidio del Otro Yo en la siguiente mañana representando el momento clímax del cuento, Armando no sabía la realidad de su situación en la que se mata a sí mismo sin conciencia o intención.

Una tarde Armando llegó cansado del trabajo, se quitó los zapatos, movió lentamente los dedos de los pies y encendió la radio. En la radio estaba Mozart, pero el muchacho se durmió. Cuando despertó el Otro Yo lloraba con desconsuelo. En el primer momento, el muchacho no supo que hacer, pero después se rehízo e insultó concienzudamente al Otro Yo. Este no dijo nada, pero a la mañana siguiente se había suicidado. (Benedetti, 2015, p. 255)

Con la perdida su humanidad, no le quedaba ni un átomo de sensibilidad, al punto, que cuando pasa enfrente de sus amigos, no lo ven. Sus amigos tuvieron que ignorar su presencia como si no existiera porque ya no le conocían. Al final, el personaje quería llorar, sentirse como ser humano, pero no podía. Toda su sensibilidad se la había llevado el Otro Yo ya que la tristeza, a pesar de su efecto negativo, es uno de los sentimientos que caracterizan a la humanidad.

Desde lejos vio que se acercaban sus amigos. Eso le lleno de felicidad e inmediatamente estalló en risotadas. Sin embargo, cuando pasaron junto a él, ellos no notaron su presencia. Para peor de males, el muchacho alcanzó a escuchar que comentaban: "Pobre Armando. Y pensar que parecía tan fuerte y saludable."

El muchacho no tuvo más remedio que dejar de reír y, al mismo tiempo, sintió a la altura del esternón un ahogo que se parecía bastante a la nostalgia. Pero no pudo sentir auténtica melancolía, porque toda la melancolía se la había llevado 
el Otro Yo. (Benedetti, 2015, p .255)

De hecho, el personaje presenta la relación entre esas dos facetas no como complementaria sino como conflictiva, lo que nos conduce al tema de la dialéctica razón-sentimientos. En otras palabras, esta relación de oposición entre el yo y el doble se concreta en la oposición entre una realidad existencial aparente y otra auténtica.

Dicho tipo de desdoblamiento, como hemos mencionado anteriormente, se presenta ahora como un recurso para explicar ese conflicto interior. El personaje siente que habitan dos facetas de sí mismo enfrentadas y opuestas dentro de él, que luchan por imponerse una a la otra.

Las referencias al desdoblamiento como un recurso que remite en esencia a la idea de oposición o enfrentamiento entre sus partes se encuentran presentes en la literatura y en el pensamiento filosófico desde sus más tempranas manifestaciones. En este sentido, Juan Bargalló señala que el tema del doble aparece en toda la literatura de Occidente como oposición de contrarios, pues, por lo general, las dos encarnaciones se comportan con un enfrentamiento entre ellas, como si no hubiera sitio en el mismo mundo para las dos, lo cual desemboca en el desenlace trágico. (Romero, 2008, p. 510)

El narrador no especifica un tiempo o un espacio ni siquiera da señales de donde se desarrolla la trama. Proponemos que él no los menciona para indicar que esto puede ocurrir en cualquier tiempo o lugar. Cada persona tiene dos personalidades: una buena que actúa con sentimientos y cuida su conciencia, y otra mala, vulgar y odiable. El ser humano elige mostrar el lado que quiere, sea bueno o malo. Él es quien determina el lado ganador de esta lucha.

En nuestro caso, el protagonista, Armando, elige el Yo vulgar e insulta al Otro Yo, lo que lleva al suicidio del último. El autor expresa con una gran figura retórica considerando.ElOtro Yo como una persona que se suicida a causa de la gran tristeza y la frustración por el mal trato de Armando.

Armando cree que la gente prefería al vulgar y que el Otro Yo impide esto. Pero, después del suicidio de éste se dio cuenta de que perdió todo, no sólo su conciencia, sino también su lado brillante sentimental, que apareció cuando se encontró con sus amigos. 
También, Benedetti hace uso de la narración lineal en toda la extensión de la obra. Establece un tiempo cronológico desde el principio hasta el final del cuento. El tiempo cronológico como se sabe es uno de los recursos de los cuentos realistas.

De todos modos, a pesar de las diferencias que se declaran entre Armando y su otro Yo, los dos juntos forman el único personaje protagonista. Por otra parte, no sabemos si el autor con toda intención o no, menciona a los amigos de Armando como otros personajes que aparecen y hacen comentarios aunque no se definen bien ni cuántos son, o quiénes son.

El narrador mantiene en tercera persona a lo largo del cuento aprovechando su perspectiva alta que sabe todo, incluso el pensamiento y el sentimiento de los personajes. Va tejiendo la historia como una verdadera araña contando con suma precisión cualquier paso que el protagonista da en su evolución hacia el clímax de la narración.

El narrador ha dejado escapar algunos detalles, pues no revela si la gente sabe la existencia de su otro Yo o no. El narrador no está sorprendido al declarar la existencia del otro yo y que el autor sufre de una especie de desdoblamiento, hasta el punto de que pensamos que todos tenemos ese otro yo.

En nuestra opinión, Benedetti con este cuento refiere a un sentido importante: debemos entender que sin nuestro Otro Yo volvemos seres sin sentimientos, como Armando que padece un dramático cambio hacia la barbarie. Luego, nos deshumanizamos, es decir, vivimos como muertos.

El tema del desdoblamiento o del doble yo, como hemos dicho anteriormente, se establece en muchas obras literarias, sobre todo, en la literatura moderna y se estudia críticamente por muchos. Igualmente, Jorge Luís Borges ha escrito un cuento titulado "El Otro" tratando el mismo recurso del otro yo, pero desde una perspectiva muy diferente.

"El Otro" plantea el tema del encuentro de la persona misma. Borges, 
después de una pequeña conversación, le dijo al joven que son la misma persona, aunque estén separados por medio siglo de vida e intenta convencerlo. Le cuenta intimidades que sólo uno mismo puede saber y por consiguiente le explica lo que le acontecerá en su vida. Borges cita una serie de acontecimientos históricos que ocurrieron en su país y en el mundo. Pero el joven niega esta idea indicando que podría tratarse de un sueño; y expone sus ideas sobre la literatura y la sociedad, que corresponden a las que Borges tuvo en la juventud. Como siempre Borges le gusta formar un estado de confusión. Al final del cuento, los dos se mienten mutuamente, diciendo que debían marcharse, y proponen reunirse al día siguiente en el mismo lugar. Ninguno aparece y Borges llega a la conclusión de que la experiencia fue real para él, pero no fue más que un sueño para el otro Borges.

Al comparar los dos cuentos: los personajes en el cuento de Borges pertenecen a dos épocas distintas. Mientras en el texto de Benedetti, los dos existen simultánea y paralelamente. Benedetti no es el narrador ni el protagonista de su cuento, mientras Borges es el protagonista, también el viejo Borges es el narrador de los acontecimientos en primera persona. Cabe mencionar que Benedetti presenta un protagonista con el nombre Armando y el narrador es un narrador omnisciente en tercera persona. En el cuento de Benedetti el narrador expone los hechos como si fueran reales y Armando y el Otro Yo mantenían en solo una persona hasta el suicidio de El Otro Yo. A cambio, los dos Borges acordaron reunirse al final del cuento, pero el joven Borges no fue.

Además, en ambos cuentos lo real y lo fantástico se juntan y se mezclan, por ejemplo, los nombres de lugares, las fechas determinadas, los nombres de personajes-llamar el protagonista con el nombre del autor y otros- son aspectos reales. También encontramos que el dualismo es fruto de esta combinación representando la base de lo fantástico:

La dimensión fantástica surge cuando un individuo, cuya conciencia personal le constituye como un sujeto único y diferente, se percibe desdoblado en otro ser que se presenta con una identidad autónoma y al mismo tiempo idéntica a la suya propia; o cuando un individuo encuentra a otro que parece un duplicado o una réplica exacta del primero o de otro individuo conocido por él (Romero, 2008, p. 252). 
Al final concluimos que el personaje del relato fantástico tiene dos posibilidades del fenómeno del doble cuando se encuentra frente a su propio doble: tipo de doble externo, si adopta una forma física y otro interno, si su manifestación es de origen psíquico a través de posesión de personalidad múltiple como en el caso del protagonista del cuento mencionado de Benedetti formando el aspecto fantástico.

\section{La interrelación de la vida y la muerte}

La muerte es un tema de mayor presencia en muchas obras literarias, y se plantea con diferentes perspectivas, López Martín intenta justificar esta presencia persistente de la muerte, especialmente, en la literatura fantástica.

La muerte, vista desde multitud de ángulos, es uno de los temas principales del cuento fantástico, dado que se identifica con la amenaza, lo inexplicable y también, por supuesto, con el ámbito de los silencios; es por excelencia el reino de lo desconocido, de lo irreductible y de la destrucción que a todos nos acecha. (Martín, 2009, p. 615)

Así, es un tema que surge frecuentemente, de muchas maneras y por muchas causas en los cuentos de Benedetti, como vemos en Lázaro donde representa la resurrección después de la muerte, uno de los factores por los que se destaca la fantasía. También, observamos la habilidad del escritor en mostrar otro tipo de muerte diferente de la física: la espiritual, una condición psíquica muy difícil representada en Como Greenwich a través de Susana, la protagonista, que vive con sentimientos de un muerto, privada de algunos de sus atributos humanos: sin una familia real, un hogar estático o una patria con el verdadero sentido, está perdida entre un pasado que no se puede recuperar y un presente desconocido en condiciones difíciles, lo que la llevó a pensar en el suicidio.

La relación entre la vida y la muerte siempre se tiñe por la inserción de las leyes de tiempo y espacio que circunscriben el transcurso de la existencia a unos códigos naturales y que, al violarse, provocan grandes inquietudes del hombre: la vida después de la muerte, lo desconocido al otro lado de la vida, el sentimiento de indefensión y de amenaza ante lo desconocido y, principalmente, ante la muerte como uno de los mayores miedos desde los tiempos más remotos. 
En el mismo contexto, muchos autores están preocupados por la relación entre el muerto y los otros vivos. López Martín cree:

Si hay un tema de particular controversia en la literatura fantástica es el de las apariciones, la revelación tangible de los espíritus que regresan de otro mundo e interfieren en éste provocando, cuando menos, una suerte de incógnitas sin solución o con solución increíble (...). Lo cierto es que ningún personaje permanece impasible después del contacto con la muerte... (Martín, 2009, p. 594)

La literatura fantástica, sobre todo, el cuento trata distintas historias donde las nociones de tiempo y espacio vitales se transgreden, lo que produce la inquietud que controla el corazón y la mente de los personajes. López Martín enumera algunos casos:

Historias sobre vampirismo y cadáveres vivientes, las almas desprendidas del cuerpo, fantasmas, espíritus y otros regresados de la muerte... todos aquellos casos que tratan de las transferencias entre el mundo de los vivos y el mundo de ultratumba han ocupado un lugar importante en la literatura fantástica desde el origen de ésta misma. También forman parte de este grupo los cuentos que incluyen alteraciones en la dimensión cronológica... y de lugar.... Incluso, aquellos relatos en los que no se da la mudanza del tiempo que debiera. (Martín, 2009, p. 573)

El relato Acaso irreparable publicado en el libro de cuentos La muerte y otras sorpresas en 1968, sigue a Sergio, el protagonista del cuento, en los aplazamientos de su vuelo durante cuatro días mientras viaja entre el hotel y el aeropuerto esperando el arreglo del avión. En el cuento encontramos una situación aparente que tiende a la normalidad, pero la situación difiere una y otra vez de un despegue de un vuelo dado que el pasajero en realidad lleva varios años muerto, realidad que se va a percibir al final del cuento.

Es lunes, 4. Rivera tenía que emprender su viaje desde Uruguay, hace escala en un país europeo, pero el vuelo se cancela postergándolo un día, se quedaba en el Hotel Internacional, piensa nítidamente en su mujer Clara y su hijo Eduardo, come en un restaurante y habla con otro viajero argentino. En martes, 5. Rivera desayuna, le trasladan al aeropuerto y a las 12 y 15 se anunció nuevo retraso de tres horas. A las 15 y 30 hay otra postergación hasta el día siguiente.

En los dos días siguientes se repitió el ritmo de los días anteriores, pero 
con un inicial rasgo sintomático de pérdida de memoria: No recordaba el cuarto nombre de los clientes que tiene que visitar: "Sólo recordó que empezaba con la letra $E$. Le fastidió tanto esa repentina laguna que decidió apagar la luz y trató de dormirse" (Benedetti, 2015, p. 273). Además, en este tramo del cuento se percata de que en veinticuatro horas no ha pensado en su mujer y continúo la pérdida de memoria, ya que no se acuerda de Clara, aunque sí de Eduardo, y de los tres nombres de los clientes se redujeron a dos: Fried y Brunelí. Más tarde, no recordó, definitivamente, ninguno de los nombres de sus clientes, aunque eso parece no afectarle. Luego, el tiempo empieza a desnaturalizarse: Benedetti acelera la desintegración del tiempo cronológico, lo que exponemos más tarde, por ejemplo, Rivera descubre un almanaque que marca el miércoles día 11 en vez de jueves día 7 (Herráez, 2000, p. 342).

Miguel Herráez opina que Benedetti es un autor de cuentos de fantasmas, que genera cierta inquietud considerando que Acaso irreparable es uno de dichos cuentos de fantasmas:

Lo aceptamos como fantasmático porque...Sergio Rivera y sus compañeros de vuelo se han convertido en seres transfísicos, aunque con una particularidad fundamental que convierte la circunstancia anecdóticamente en respuesta al género: no es Rivera y el resto de espíritus quienes regresan al mundo de los vivos, sino los vivos quienes se les aparecen a ellos en ese mundo de vivos pero ocupado por los muertos: la terminal del aeropuerto. (Herráez, 2000, p. 341)

Entre las particularidades más características de la literatura fantástica se destaca la mezcla de lo real y lo fantástico, presentar lo fantástico como algo habitual y cotidiano. Por ello, pensamos que la realidad literaria de este cuento existe dentro de los límites del realismo mágico que explica, por ejemplo el conformismo del protagonista, propio de un muerto, el olvido progresivo de sus familiares y negocios y por último, la destrucción temporal que comprobamos al no coincidir las fechas de los calendarios con su creencia temporal. Todo ello confirma la identidad de Rivera, la de un muerto.

Benedetti presenta una ordenación lineal al principio que luego se siente incomprendida de los acontecimientos hasta el punto final en el que descubrimos que todos estos acontecimientos ocurren desde años y el 
protagonista ya está muerto. En cuanto al tiempo:

Esta metamorfosis del protagonista se lleva a cabo en un primer momento ante un fondo obviamente cronológico y realista de postergaciones del vuelo: «24 horas-martes 5 'en principio' para las 11 y 30 -12 y 15 - nueva postergación probablemente de tres horas mañana a las 12 y $30 »$, lo que corresponde a una espera de lunes día 5 hasta miércoles día 7 de un mes cualquiera. (Weitzdörfer, 2000, p. 356)

La estructura del tiempo pierde gradualmente el hilo. Ya no queda armonizado con el sentimiento temporal de Rivera como persona natural, liberado de la lógica. Así que las indicaciones de los aplazamientos del vuelo pasan cada vez más imprecisas: "mañana, a hora sin determinar" (Benedetti, 2015, p. 275). Entonces, empiezan a tambalearse por lo visto hasta el orden de días y meses, "en vez de jueves 7, (el almanaque) marcaba miércoles 11" (Benedetti, 2015, p. 275). Según el esquema temporal del principio del cuento, el día 11 tendría que caer en lunes; por eso, sólo se puede pertenecer a otro mes o de otro año. Después de esta confusión, la hoja del almanaque (lunes 7) que cae en manos de Rivera se enfrenta con indiferencia y más tarde ya no tendrá ningún sentido, "La fecha... era tan descabellada, que decidió no darle importancia" (Benedetti, 2015, p .277).

Por eso, no le puede interesar mucho el estado actual del avión averiado en la pista de despegue, rodeado por los técnicos como un enfermo en estado grave por el equipo de médicos y enfermeras donde podemos entender el título del cuento «Acaso irreparable» parece corroborar esa situación difícil:

De vez en cuando una voz, siempre femenina, anunciaba la llegada de un avión, la partida de otro. Nunca, por supuesto, del vuelo 914 de LCA, cuyo paralizado, invicto avión seguía en la pista, cada vez más rodeado de mecánicos en overalls, largas mangueras, jeeps que iban y venían trayendo o llevando nuevos operarios, o tornillos u órdenes. (Benedetti, 2015, p.276).

De repente, una vez más, su nueva existencia roza su vida anterior, cuando Sergio percibe la presencia de su hijo, ahora ya mayor, con una chica en el aeropuerto ante el fondo acústico de los usuales anuncios de los altavoces. Los dos jóvenes habían venido a Europa para una estancia más larga en Viena y Nuremberg respectivamente. Durante la escala en este aeropuerto intercambian sus direcciones. Cuando la chica se entera 
de que Eduardo Rivera se quedará un año entero en Viena exclama, “¿Y tu viejo no protesta?" (Benedetti, 2015, p .277). A esta pregunta sigue el final sorprendente del cuento:

El muchacho empezó a decir algo. Desde su sitio, Sergio no pudo entender las palabras porque en ese preciso instante el parlante (la misma voz femenina de siempre, aunque ahora extrañamente cascada) informaba: «LCA comunica que, en razón de desperfectos técnicos, ha resuelto cancelar su vuelo 914 hasta mañana, en hora a determinar». Sólo cuando el anuncio llegó a su término, la voz del adolescente fue otra vez audible para Sergio: «Además, no es mi viejo sino mi padrastro. Mi padre murió hace años, ¿sabés?, en un accidente de aviación. (Benedetti, 2015, p.277)

\section{Conclusión}

Podemos concluir que Benedetti combina perfectamente lo real y lo fantástico en los dos cuentos estudiados mezclando los recursos del realismo y de la fantasía: en el cuento El otro yo donde aparece esta combinación como la linealidad del tiempo y la descripción de la normalidad de la vida de Armando, el protagonista que tiene dos personalidades que no son diferentes solo en sus cualidades, sino también en sus actos hasta el punto de que una murió antes de la otra. Pues el escrito nos presenta una perspectiva particular del tema del doble.

En el cuento de Acaso irreparable que empieza por una narración normal de un viaje donde menciona el autor lugares reales, fechas concretas que son marcas de narraciones reales, el autor utiliza dos características del realismo mágico: primero, el tiempo irregular donde el pasado y el presente se imbrican puesto que existe poca distancia entre ellos. Al principio del cuento se narra los acontecimientos de los cuatro días del viaje del protagonista y al final del cuento descubrimos que su hijo es el que pasa por el aeropuerto acompañando a una chica que su padre murió desde años. Así veremos que como la muerte del padre, está entrelazado con el tiempo de su hijo que ya ha crecido. En el mismo contexto, el tiempo tiene una peculiar presencia en el realismo mágico. El tiempo no es lineal a lo largo del cuento, pero, nos enfrentamos con un tiempo irracional, o sea, hay saltos temporales, ya que dentro de la narración se unen diferentes niveles espacio-temporales, donde los recuerdos o alusiones se mezclan con el momento real narrado. 
El segundo aspecto estudiado que es la percepción de, la muerte, se observa que ya no es elemento tan terrible y se ve como un fenómeno natural más allá del horror o la tristeza que la caracterizan. La muerte es concebida como parte de la vida, e incluso es complicado establecer los límites entre la vida y la muerte, de acuerdo con el realismo mágico este cuento se puede pertenecer a la literatura de fantasmas, en la que no se puede estar seguros de si los muertos eran visibles a los vivos, o, su presencia era solo espiritual en la vida de los vivos.

\section{Bibliografía}

Bay, C. A., Azuar, R. M.\& Soler, C. R. (Eds.). (2000). Mario Benedetti: Inventario cómplice. Biblioteca Virtual Miguel de Cervantes. Versión electrónica disponible en http://www.cervantesvirtual.com/obra/mario-benedettiinventario-complice--0/

Benedetti, M. (2015). Cuentos completos. Alfaguara.

Cecilia, J. H. (2000). Estética y pragmática del relato fantástico. Ediciones de la Universidad de Castilla-La Mancha.

Martín, L. L. (2009). Formación y desarrollo del cuento fantástico hispanoamericano en el siglo xix, tesis doctoral, Universidad Autónoma de Madrid, Facultad de Filosofía y Letras.

Romero, C.C, M., (2008). Realidad y ficción en la obra de Carmen Martín Gaite, tesis doctoral, Universidad de Extremadura. 